12

\title{
Кулоновские плазмон-экситоны в планарных наноструктурах металл-полупроводник
}

\author{
(C) В.А. Кособукин \\ Физико-технический институт им. А.Ф. Иофрфе РАН, \\ Санкт-Петербург, Россия \\ E-mail: Vladimir.Kosobukin@mail.ioffe.ru
}

Поступила в Редакцию 26 ноября 2020 г.

В окончательной редакции 26 ноября 2020 г.

Принята к публикации 27 ноября 2020 г.

\begin{abstract}
Представлена теория кулоновских (нерадиационных) плазмон-экситонов в полупроводнике с близко расположенными квантовой ямой и ультратонкой металлической пленкой. Сформулированы уравнения движения для волн поляризации поверхностных плазмонов и квазидвумерных экситонов с учетом их кулоновского взаимодействия. В рамках модели связанных гармонических осцилляторов решены задачи о вынужденных кулоновских плазмонных, экситонных и плазмон-экситонных возбуждениях при наличии дипольной вынуждающей силы. Для плазмон-экситонов вычислена константа связи, исследованы оптические спектры плазмон-экситонов и определены относительные вклады плазмонной и экситонной компонент в нормальные моды. Показано, что вблизи резонанса между плазмонами и экситонами спектр плазмонэкситонных возбуждений состоит из двух пиков, поведение которых при похождении резонанса имеет характер антипересечения (расталкивания их частот).
\end{abstract}

Ключевые слова: плазмоны, экситоны, кулоновская связь, плазмон-экситоны, волны поляризации, антипересечение.

DOI: 10.21883/FTT.2021.04.50720.248

\section{1. Введение}

Плазмоны в металлах и экситоны в полупроводниках представляют собой коллективные электронные возбуждения со спектром в видимом диапазоне [1,2]. В наноструктурах металл-полупроводник и метаматериалах плазмоны и экситоны могут сосуществовать и взаимодействовать друг с другом [3,4]. Сравнительно давно был поставлен вопрос о гашении экситонов плазмонами вблизи поверхности металла [5,6]. В дальнейшем выяснилось, что эффекты резонансного взаимодействия плазмонов и экситонов разнообразны: плазмоны могут как гасить излучение экситонов [6-8], так и усиливать его [7-12]. По этой причине оптические плазмон-экситонные возбуждения представляют значительный научный и практический интерес. Весьма важно установить характер связи между плазмонами и экситонами, если согласно общим представлениям под связью понимать влияние одного возбуждения на другое и наоборот [13].

К настоящему времени опубликовано много работ, посвященных взаимодействию молекулярных экситонов с поверхностными плазмонами и плазмонными поляритонами. Изучалось расщепление спектра поверхностных плазмонных поляритонов при резонансе с оптическими переходами в тонких переходных слоях [14-16]. Интерес к молекулярным системам, в том числе к органическим полупроводникам, обусловлен сильной связью их экситонов с плазмонами, которая выражается в наблюдаемом большом расщеплении Раби оптических спектров [17-20]. Было установлено, что взаимодействие молекулярных экситонов и плазмонов приводит к образованию их связанных состояний [7,17-20], существенно ускоряет спонтанное излучение экситонов $[8,9]$, способствует электромагнитному энергопереносу в отсутствие электронного переноса [12,21] и т. п. Для молекулярных экситонов Френкеля следует также особо отметить работы, в которых изучались их гибридные моды с экситонами большого радиуса (экситонами Ванье) [22,23].

Взаимодействие экситонов большого радиуса с плазмонами исследовалось экспериментально в некоторых наноструктурах металл-полупроводник. Преимущественно обсуждалась связь оптически активных плазмонов с экситонами квантовых точек $[7,8]$ или квантовых ям [24,25]. Для структур с квантовыми ямами теоретически предсказывается двухпиковая структура плазмонэкситонных оптических спектров. Это относится к рассеянию света наночастицей благородного металла вблизи квантовой ямы [26,27], к отражению света от ямы и слоя частиц [28], к распространению поляритонов в сверхрешетке квантовых ям и слоев металлических наночастиц [29]. Поведение пиков при сближении частот плазмона и экситона имеет признаки антипересечения (расталкивания частот) вблизи резонанса [30].

Кроме излучательных плазмонов и экситонов, значительный физический интерес представляют их нерадиационные аналоги. В системах пониженной размерности нерадиационные (кулоновские) парциальные возбуждения и их смешанные моды имеют характер волн поляризации. Эти волны распространяются в направлениях трансляционной симметрии структуры и локализованы 
в направлениях ограничения движения. Кулоновские возбуждения могут играть важную роль в люминесценции, в рассеянии света, в ближнеполевой оптике, в безызлучательном переносе электронных возбуждений и т. п., но их систематическому изучению не уделялось достаточного внимания.

Цель настоящей работы - построение теории взаимодействия нерадиационных экситонов большого радиуса и поверхностных плазмонов в наноструктурах с близко расположенными квантовой ямой и слоем металла. Сформулированы уравнения динамики для поляризации квазидвумерных экситонов и плазмонов при их кулоновском взаимодействии и наличии вынуждающей силы. Исследованы спектроскопические эффекты, обусловленные связью нерадиационных („темных“) экситонов с поверхностными плазмонами. Содержание работы заключается в следующем. Постановка задачи дана в разд. 2, математическая модель сформулирована в разд. 3. На этой основе динамические уравнения для поляризации построены в разд. 4 без учета плазмонэкситонного взаимодействия и в разд. 5 - с учетом взаимодействия. Спектры плазмон-экситонов проанализированы в разд. 6 в пренебрежении их диссипативным затуханием, а в разд. 7 - с учетом затухания.

\section{2. Постановка задачи}

Для изучения плазмон-экситонов в наноструктурах металл-полупроводник используем модель, показанную на рис. 1. Рассматривается полупроводник с близко расположенными квантовой ямой и металлической пленкой, причем ширина ямы $l$, толщина пленки $L$ и расстояние $h$ между их центральными плоскостями имеют нанометровый масштаб. Предметом изучения служат нерадиационные плазмон-экситоны, образованные при взаимодействии поверхностных плазмонов пленки с квазидвумерными (квази-2D)-экситонами ямы. В пренебрежении электромагнитным запаздыванием эти возбуждения являются кулоновскими по терминологии [15].

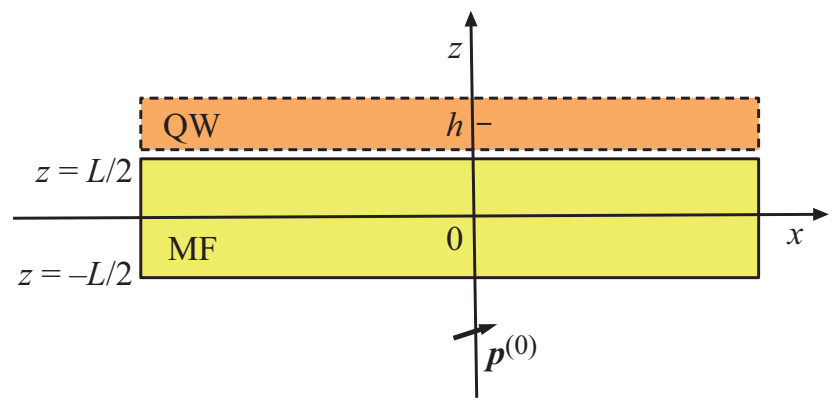

Pис. 1. Геометрия задачи: металлическая пленка (MF) толщиной $L(|z|<L / 2)$, квантовая яма $(\mathrm{QW})$ со средней плоскостью $z=h>L / 2$ и дипольный осциллятор при $z=z_{0}<-L / 2$. Фоновая диэлектрическая проницаемость равна $\varepsilon_{1}=\varepsilon_{b}$ при $|z|>L / 2$ и $\varepsilon_{2}=\varepsilon_{\infty}$ при $|z|<L / 2$, формула (1).

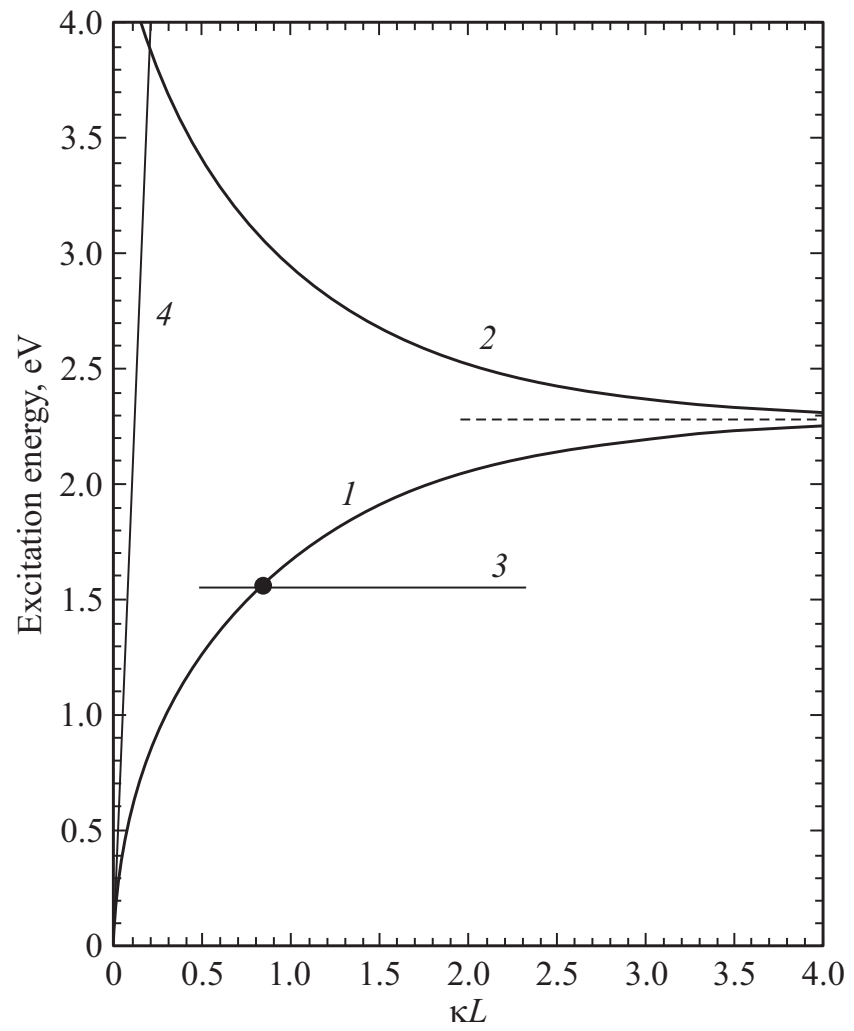

Рис. 2. Зависимость от $\kappa L$ энергий $\hbar \omega_{-}(1)$ и $\hbar \omega_{+}(2)$ поверхностных плазмонов в пленке $\mathrm{Ag}$ толщиной $L$ и квази-2Dэкситонов $\hbar \omega_{0}(3)$ в квантовой яме GaAs/AlGaAs. B точке пересечения плазмонной $\omega_{-}(\kappa)$ и экситонной $\omega_{0}$ ветвей выполняется условие $\omega_{-}(\kappa)=\omega_{0}$ плазмон-экситонного резонанса. Прямая 4 показывает при $L=3 \mathrm{~nm}$ границу светового конуса $\omega=\kappa c / \sqrt{\varepsilon_{b}}$, вне которого находятся нерадиационные возбуждения с $\kappa>\sqrt{\varepsilon_{b}} \omega / c$. Вычислено при $\hbar \omega_{p}=9.45 \mathrm{eV}, \varepsilon_{\infty}=4.3$ для $\mathrm{Ag}$ [32] и $\hbar \omega_{0}=1.55 \mathrm{eV}, \varepsilon_{b}=12.5$ для GaAs.

Модельная структура (рис. 1) трансляционно инвариантна по $\boldsymbol{\rho}=(x, y)$ и изотропна в плоскостях $z=$ const. Невзаимодействующие (парциальные) поверхностные плазмоны в пленке и квази-2D-экситоны в яме представляют собой двумерные волны диэлектрической поляризации $\mathbf{P}(\mathbf{r}, t)=\mathbf{P}(z, \boldsymbol{\kappa}, \omega) e^{i \kappa \boldsymbol{\rho}-i \omega t}$. Их законы дисперсии изотропны по направлениям волнового вектора $\boldsymbol{\kappa}=\left(\kappa_{x}, \kappa_{y}\right)$ в плоскости $x y$, причем для кулоновских (квазистатических) мод $|\boldsymbol{\kappa}| \gg k_{0}=\omega / c$, где $\omega-$ частота, $c$ - скорость света. Электрическое поле, связанное с волной поляризации $\mathbf{P}(z, \boldsymbol{\kappa}, \omega)$, затухает по закону $E(z, \boldsymbol{\kappa}) \sim e^{-\kappa|z|}$ при увеличении расстояния $|z|$ от планарной наноструктуры. Поле $\mathbf{E}(z, \boldsymbol{\kappa})$ поляризационной моды с $\boldsymbol{\kappa}=\kappa \mathbf{e}_{x}$ имеет продольную $x$ и поперечную $z$ компоненты, а $y$-компонента, имеющая малость $\sim\left(k_{0} / \kappa\right)^{2} \ll 1$ в квазистатике, пренебрежимо мала.

В металлической пленке $(|z|<L / 2)$, заключенной между диэлектрическими средами, поверхностные плазмоны имеют две дисперсионные ветви в зависимости от $\boldsymbol{\kappa}$. В случае одинаковых сред ветви $\omega_{\mp}(\boldsymbol{\kappa})$ показаны как 1 и 2 на рис. 2, где индексы ₹ соответствуют 
модам плазмонов, симметричной и антисимметричной относительно плоскости $z=0$. Сказанное о квазистатических модах плазмонов с $\kappa \gg k_{0}$ справедливо, если $L$ превышает толщину нескольких атомных слоев [31]. При учете электромагнитного запаздывания спектры $\omega_{\mp}(\boldsymbol{\kappa})$ перестраиваются только в области $\kappa \gtrsim k_{0}$ вблизи фотонной прямой 4, где плазмоны трансформируются в нерадиационные плазмон-поляритоны [2].

В квантовой яме шириной $l \ll a_{B}$, где $a_{B}-$ боровский радиус экситона в материале ямы, квази-2D-экситоны с $\kappa \gg k_{0}$ не взаимодействуют со светом. В нерадиационной области $\left(\kappa \gg k_{0}\right)$ экситоны характеризуются теми же микроскопическими зонными параметрами, что вычислялись для радиационных квази-2D-экситонов [33-35].

Для взаимодействия экситона и плазмона их законы дисперсии должны удовлетворять условию $\omega_{\text {plasmon }}(\boldsymbol{\kappa})=\omega_{\text {exciton }}(\boldsymbol{\kappa})$ (см. пересечение ветвей 1 и 3 на рис. 2). В случае кулоновских парциальных мод смешанная плазмон-экситонных мода не может возбуждаться световыми волнами, имеющими волновые числа $\sim k_{0}$. Для возбуждения „темных“ мод необходимы источники излучения с широким пространственным спектром $\kappa \gg k_{0}$ в плоскости $x y$ наноструктуры, такие как квазиточечные ближнеполевые зонды, наночастицы, атомные агрегаты и т.д.

\section{3. Основные уравнения}

В модели, схематически представленной на рис. 1, фоновая (при отсутствии плазмонной и экситонной поляризации) диэлектрическая проницаемость равна

$$
\begin{gathered}
\varepsilon(z)=\varepsilon_{1}[1-v(z)]+\varepsilon_{2} v(z), \\
v(z)=\theta\left(z+\frac{L}{2}\right)-\theta\left(z-\frac{L}{2}\right) .
\end{gathered}
$$

Здесь $\varepsilon_{1}$ и $\varepsilon_{2}-$ положительные постоянные, а функция $\theta(z)$ равна нулю при $z<0$ и единице при $z>0$. Далее считаем, что область $|z|>L / 2$ занята полупроводником с фоновой проницаемостью $\varepsilon_{1}=\varepsilon_{b}$, а область $|z|<L / 2$ занята металлом с $\varepsilon_{2}=\varepsilon_{\infty}$.

На фоне статической проницаемости (1) возбуждаются плазмонная $\mathbf{P}^{(1)}$ и экситонная $\mathbf{P}^{(2)}$ поляризации, которым далее соответствуют верхние индексы 1 и 2. Поляризационные бозе-возбуждения обоих типов можно описывать как квантовые [1] или классические (при больших числах заполнения состояний) [2,15]. В данной работе в приближении классической электродинамики решается задача о колебаниях гармонических осцилляторов с кулоновской связью, роль которых выполняют поляризации $\mathbf{P}^{(1)}$ и $\mathbf{P}^{(2)}$.

Плазмоны возбуждаются в пленке металла толщиной $L$, содержащей электронный газ с однородной плотностью $n_{0} v(z)=$ const. Для волны плазмонной поляризации с вектором $\boldsymbol{\kappa}$ в пленке локальное уравнение линейного отклика имеет вид

$$
-\left(\omega^{2}+i \omega \gamma\right) \mathbf{P}^{(1)}(z, \boldsymbol{\kappa}, \omega)=\frac{\omega_{p}^{2}}{4 \pi} v(z) \mathbf{E}(z, \boldsymbol{\kappa}, \omega) .
$$

Здесь $\omega_{p}=\left(4 \pi n_{0} e^{2} / m\right)^{1 / 2}-$ плазменная частота, $e, m$ и $\gamma$ - заряд, эффективная масса и обратное время релаксации электронов в металле, $v(z)$ дается формулой (1) и $\mathbf{E}$ - полное электрическое поле.

Для поляризации основного $1 s$-состояния квази-2Dэкситона квантовой ямы, следуя теории [35] в приближении эффективной массы, находим нелокальное материальное соотношение

$$
\begin{aligned}
\left(\omega_{0}^{2}(\kappa)\right. & \left.-\omega^{2}-i \omega \Gamma\right) P_{\alpha}^{(2)}(z, \kappa, \omega)=\Omega_{(\alpha)}^{2} \psi(z-h) \\
& \times \int d z^{\prime} \psi\left(z^{\prime}-h\right) E_{\alpha}\left(z^{\prime}, \boldsymbol{\kappa}, \omega\right) .
\end{aligned}
$$

Здесь $\omega_{0}(\kappa)=\omega_{0}+\hbar \kappa^{2} /(2 M)-$ закон дисперсии экситона с частотой $\omega_{0}$, учитывающей размерное квантование носителей, с трансляционной массой $M$ и параметром диссипативного затухания Г. Свойства огибающей $\psi(z-h)$ волновой функции основного состояния квази-2D-экситона при совпадающих координатах электрона и дырки в яме со средней плоскостью $z=h$ обсуждаются в Приложении А.

Поляризационный отклик (3) квази-2D-экситона на электрическое поле определяется константой $\Omega_{(\alpha)}^{2}=\omega_{0} \Gamma_{e,(\alpha)}$, где индекс $(\alpha)$ обозначает ось поляризации экситона. С учетом теории [35] полагаем $\Gamma_{e,(\alpha)}=p_{(\alpha)} \varepsilon_{b} \omega_{L T} /(2 \pi)$, где $p_{(\alpha)}>0$ для оси $\alpha$, в направлении которой экситон оптически активен, а частота $\omega_{L T}$ выражается через межзонный матричный элемент оператора импульса $[35,36]$. В случае простой (изотропной) зоны $\Omega_{(\alpha)}=\Omega$. Для прямозонных полупроводников со структурой $\mathrm{ZnS}$ основные состояния экситонов $e 1-h h 1$ тяжелой и $e 1-l h 1$ легкой дырок оптически активны в плоскости $x y$ квантовой ямы, а экситон $e 1-l h 1$ имеет также ненулевую силу осциллятора по нормали $z$ к яме $[35,36]$.

Для дальнейшего важно, что в согласии с [33-36] для всех перечисленных экситонов $\Gamma_{e,(x)}=\Gamma_{e,(y)}$, при этом $\Gamma_{e,(z)}=0$ для экситона $e 1-h h 1$ и $\Gamma_{e,(z)}>\Gamma_{e,(x)}$ для экситона $e 1-l h 1$. Величины $\Gamma_{e,(\alpha)}=\Gamma_{e}$ для касательных $(\alpha=x, y)$ компонент поляризации связаны с параметром радиационного затухания квази-2D-экситона

$$
\begin{aligned}
\Gamma_{0} & =\Gamma_{e} \frac{2 \pi \omega_{0}}{c \sqrt{\varepsilon_{b}}}\left[\int d z \psi(z) \cos \left(\frac{\sqrt{\varepsilon_{b}} \omega_{0} z}{c}\right)\right]^{2} \\
& \approx \omega_{L T} \frac{\omega_{0} \sqrt{\varepsilon_{b}}}{c}\left(\int d z \psi(z)\right)^{2},
\end{aligned}
$$

который вводится при нормальном падении света на квантовую яму шириной $l \ll c /\left(\sqrt{\varepsilon_{b}} \omega_{0}\right)[34,35]$. Согласно формуле (А2) из Приложения А квадрат интеграла в (4) определяет эффективную ширину $l_{\text {eff }} \sim a_{B}>l$ 
в противоположность „физической“ ширине квантовой ямы $l$. В рамках микроскопической теории параметр $\Gamma_{0}$ численно исследовался для квантовых ям GaAs/AlGaAs в работе [37]. Этот параметр определяет коэффициент нормального отражения света

$$
R(\omega)=\frac{\Gamma_{0}^{2}}{\left(\omega_{0}-\omega\right)^{2}+\left(\Gamma+\Gamma_{0}\right)^{2}},
$$

который вычисляется на основе представления (3) и определяется из оптического эксперимента [37]. Найденный таким образом параметр $\Gamma_{0}$ может служить для экспериментальной оценки величин $\Gamma_{e}$ и $\Omega_{(\alpha)}$, входящих в (3), (4).

Заметим, что соотношение (3) для $\mathbf{P}^{(2)}$ получено на основе результатов квантовой теории применительно к модели классического осциллятора, поэтому в (3) входят квадраты частот, как и в выражение (2) для $\mathbf{P}^{(1)}$. Как следствие, параметры затухания, которые мы используем для экситонов, вдвое больше, чем в квантовой задаче. Чтобы получить для полей поляризации уравнения движения, зависящие от времени $t$, следует сделать в (2), (3) и далее замены

$$
\begin{gathered}
-\left(\omega^{2}+i \omega \gamma\right) \rightarrow \frac{d^{2}}{d t^{2}}+\gamma \frac{d}{d t}, \\
-\omega^{2}-i \omega \Gamma+\omega_{\alpha}^{2} \rightarrow \frac{d^{2}}{d t^{2}}+\Gamma \frac{d}{d t}+\omega_{\alpha}^{2}
\end{gathered}
$$

в соответствии с преобразованием Фурье $P_{\alpha}^{(n)}(z, \kappa, \omega) \rightarrow$ $P_{\alpha}^{(n)}(z, \kappa, t)$.

В общем случае будем рассматривать поляризацию

$$
\mathbf{P}(\mathbf{r}, t)=\mathbf{P}^{(0)}+\mathbf{P}^{(1)}+\mathbf{P}^{(2)}
$$

которая кроме $\mathbf{P}^{(1)}$ и $\mathbf{P}^{(2)}$ включает вклад $\mathbf{P}^{(0)}$ внешнего источника. Для последнего принимаем $\mathbf{P}^{(0)}(\mathbf{r}, t)=\mathbf{p}^{0}(t) \delta(\boldsymbol{\rho}) \delta\left(z-z_{0}\right), \quad$ где $\quad \mathbf{p}^{0}(t) \quad$ - дипольный момент осциллятора, расположенного в точке $\mathbf{r}=\left(0,0, z_{0}\right), z_{0}=-\left|z_{0}\right|<-L / 2$ (рис. 1). При любом $\boldsymbol{\kappa}$ имеем

$$
\mathbf{P}^{(0)}(z, \boldsymbol{\kappa}, \omega)=\mathbf{p}^{0}(\omega) \delta\left(z-z_{0}\right) .
$$

Пусть для поляризации с $n=0,1$ или 2 из выражения (5) условие $\mathbf{P}^{(n)}\left(z^{\prime}, \boldsymbol{\kappa}\right) \neq 0$ выполняется в области $z^{\prime} \in v_{n}^{\prime}$. Поляризация $\mathbf{P}^{(n)}$ с $\boldsymbol{\kappa}=\kappa \mathbf{e}_{x}$ порождает в точке $z$ слоистой среды компоненты

$$
E_{\alpha}^{(n)}(z, \kappa, t)=\sum_{\beta} \int_{v_{n}^{\prime}} d z^{\prime} g_{\alpha \beta}\left(z, z^{\prime}, \kappa\right) P_{\beta}^{(n)}\left(z^{\prime}, \kappa, t\right)
$$

полного электрического поля, которое входит в правые части выражений (2) и (3). В формуле (7) $g_{\alpha \beta}\left(z, z^{\prime}, \kappa\right)-$ тензорные компоненты функции Грина, вычисленные в квазистатическом приближении для среды с фоновой проницаемостью (1) в отсутствие поляризации (5). Необходимый для решения нашей задачи набор функций $g_{\alpha \beta}\left(z, z^{\prime}, \kappa\right)$ с индексами $\alpha$ и $\beta$, равными $x$ и $z$, приведен в Приложении В.

С учетом формул (5) и (7) из уравнения (2) для поляризации плазмонов с заданными $\kappa, \omega$ получаем

$$
\begin{aligned}
-\left(\omega^{2}\right. & +i \omega \gamma) P_{\alpha}^{(1)}(z)=\frac{\omega_{p}^{2}}{4 \pi} v(z) \\
& \times \sum_{n=0,1,2} \sum_{\beta} \int_{v_{n}^{\prime}} d z^{\prime} g_{\alpha \beta}\left(z, z^{\prime}\right) P_{\beta}^{(n)}\left(z^{\prime}\right),
\end{aligned}
$$

В правой части выражения (8) член с $n=1$ учитывает собственное электрическое поле (7) плазмонов, а члены с $n=0$ и 2 выражают поля внешнего источника и экситонов.

Аналогично из соотношения (3) с учетом (5), (7) находим

$$
\begin{aligned}
& \left(\omega_{0}^{2}(\kappa)-\omega^{2}-i \omega \Gamma\right) P_{\alpha}^{(2)}(z)=\Omega_{(\alpha)}^{2} \psi(z-h) \\
& \quad \times \int_{v_{2}^{\prime}} d z^{\prime} \psi\left(z^{\prime}-h\right) \sum_{n=0,1,2} \sum_{\beta} \int_{v_{n}^{\prime \prime}} d z^{\prime \prime} g_{\alpha \beta}\left(z^{\prime}, z^{\prime \prime}\right) P_{\beta}^{(n)}\left(z^{\prime \prime}\right) .
\end{aligned}
$$

В уравнениях (8) и (9) члены, связанные с поляризациями плазмонов $\mathbf{P}^{(1)}$ и экситонов $\mathbf{P}^{(2)}$, перенесем в левые части, а в правых частях оставим члены с полем $\mathbf{E}^{(0)}$ вида (7), которое создается поляризацией (6) внешнего диполя. Это дает систему четырех неоднородных интегральных уравнений для поляризации взаимодействующих кулоновских плазмонов и экситонов с $\alpha=x, z$ во внешнем поле.

\section{4. Поверхностные плазмоны и квазидвумерные экситоны}

Вначале обсудим по-отдельности задачи о плазмонах и экситонах при наличии внешнего источника (6). Эти возбуждения, парциальные по отношению к плазмонэкситонам, могут проявляться самостоятельно в ближнеполевой оптике и в процессах рассеяния света.

4.1. Поверхностные плазмоны. Уравнения дисперсии для поверхностных плазмонных поляритонов с волновым вектром $\boldsymbol{\kappa}=\kappa \mathbf{e}_{x}$ в пленке металла толщиной $L$ (рис. 1) с проницаемостью $\varepsilon$ имеют вид

$$
\frac{\varepsilon}{\varepsilon_{1}}+\sqrt{\frac{1-\varepsilon\left(k_{0} / \kappa\right)^{2}}{1-\varepsilon_{1}\left(k_{0} / \kappa\right)^{2}}} \operatorname{th}^{\mp 1}\left(\frac{\kappa L}{2} \sqrt{1-\varepsilon\left(\frac{k_{0}}{\kappa}\right)^{2}}\right)=0
$$

Примем $\varepsilon(\omega)=\varepsilon_{\infty}-\omega_{p}^{2} /\left(\omega^{2}+i \omega \gamma\right)$ для металла и пренебрежем электромагнитным запаздыванием $\left(k_{0} \ll \kappa\right)$. Тогда для поверхностных плазмонов в пленке из урав- 
нений (10) находим две дисперсионные ветви $(\gamma=0)$ :

$$
\omega_{\mp}(\kappa)=\frac{\omega_{p}}{\sqrt{\Delta_{\mp}(\kappa)}}, \quad \Delta_{\mp}(\kappa)=\varepsilon_{\infty}+\varepsilon_{b} \operatorname{th}^{\mp 1}\left(\frac{\kappa L}{2}\right) .
$$

Ветви $\omega_{\mp}(\kappa)$, рассчитанные по формулам (11) с фоновыми проницаемостями $\varepsilon_{1}=\varepsilon_{b}$ в GaAs и $\varepsilon_{2}=\varepsilon_{\infty}$ в тонкой пленке $\mathrm{Ag}\left(L \ll 1 / k_{0}\right)$ показаны кривыми 1 и 2 на рис. 2 в зависимости от $\kappa L$.

Собственные векторы для мод плазмонной поляризации с частотами $\omega_{\mp}(\kappa)$ равны

$$
\begin{gathered}
\pi^{-}(z, \kappa)=\mathbf{e}_{x} \operatorname{ch} \kappa z-i \mathbf{e}_{z} \operatorname{sh} \kappa z, \\
\pi^{+}(z, \kappa)=\mathbf{e}_{x} \operatorname{sh} \kappa z-i \mathbf{e}_{z} \operatorname{ch} \kappa z
\end{gathered}
$$

в пленке $(|z|<L / 2)$. Следует заметить, что в модах $\boldsymbol{\pi}^{\mp}(z, \kappa)$ электронная плотность $\rho_{\mp}(z, \kappa) \exp (i \kappa x)$ с

$$
\rho_{\mp}(z, \kappa) \sim \delta(z+L / 2) \pm \delta(z-L / 2)
$$

локализована на поверхностях $z=\mp L / 2$. В моде $\pi^{-}$ распределение плотности заряда симметрично по $z$, а в моде $\boldsymbol{\pi}^{+}$антисимметрично. Такую же симметрию по $z$ в модах (12) имеет касательная $x$-компонента электрического поля $\mathbf{E}$, которое соответственно квазипродольно и квазипоперечно по отношению к волновому вектору $\boldsymbol{\kappa}=\kappa \mathbf{e}_{x}$.

Для плазмонной поляризации $\mathbf{P}^{(1)}$ в пленке используем следующее разложение по собственным модам (12):

$$
\begin{gathered}
P_{x}^{(1)}=V_{-} \operatorname{ch} \kappa z+V_{+} \operatorname{sh} \kappa z, \\
P_{z}^{(1)}=-i\left(V_{-} \operatorname{sh} \kappa z+V_{+} \operatorname{ch} \kappa z\right),
\end{gathered}
$$

где $V_{\mp}(\kappa, \omega)-$ комплексные амплитуды. Эти компоненты поляризации удовлетворяют соотношению $\operatorname{div} \mathbf{P}^{(1)}=0$, или

$$
i \kappa P_{x}^{(1)}+\frac{d P_{z}^{(1)}}{d z}=0
$$

Подставим выражения (6), (13) и функции Грина (B1)-(B8) из Приложения В в уравнение (8) с $\mathbf{P}^{(2)}=0$. Выполнив интегрирование, получаем

$$
\begin{aligned}
& {\left[\omega_{-}^{2}(\kappa)-\tilde{\omega}^{2}\right] V_{-} \operatorname{ch} \kappa z+\left[\omega_{+}^{2}(\kappa)-\tilde{\omega}^{2}\right] V_{+} \operatorname{sh} \kappa z} \\
& =\frac{\kappa}{2}\left(\omega_{-}^{2}(\kappa) \frac{\operatorname{ch} \kappa z}{\operatorname{sh}(\kappa L / 2)}-\omega_{+}^{2}(\kappa) \frac{\operatorname{sh} \kappa z}{\operatorname{ch}(\kappa L / 2)}\right) e^{-\kappa\left(\left|z_{0}\right|-L / 2\right)} p_{+}^{0} .
\end{aligned}
$$

Здесь $\tilde{\omega}^{2}=\omega^{2}+i \omega \gamma, \omega_{\mp}(\kappa)-$ частоты (11) мод $V_{\mp}(\kappa)$ и

$$
p_{+}^{0}(\omega)=p_{x}^{0}+i p_{z}^{0} .
$$

Динамическая матрица в уравнении (15) диагональна для мод $(12)$ с частотами $\omega_{\mp}(\kappa)$.
Умножим выражение (15) на ch $\kappa z$ или sh $\kappa z$ и проинтегрируем по $|z|<L / 2$ с учетом ортогональности базисных функций (12). В результате для амплитуд поверхностных плазмонов, возбуждаемых в пленке внешним источником, находим

$$
V_{\mp}(\kappa, \omega)=p_{+}^{0} \frac{\kappa}{2} \frac{\omega_{\mp}^{2}(\kappa)}{\omega_{\mp}^{2}(\kappa)-\omega^{2}-i \omega \gamma} f_{\mp}(\kappa) e^{-\kappa\left(\left|z_{0}\right|-L / 2\right)},
$$

где $f_{-}(\kappa)=1 / \operatorname{sh}(\kappa L / 2)$ и $f_{+}(\kappa)=-1 / \operatorname{ch}(\kappa L / 2)$.

4.2. Квази-2D-экситоны. Рассмотрим теперь задачу о нерадиационных („темных“) экситонах квантовой ямы, которая находится в полупроводнике с $\varepsilon_{1}=\varepsilon_{b}$ вблизи слоя $|z|<L / 2$ с фоновой проницаемостью $\varepsilon_{2}=\varepsilon_{\infty}$ (рис. 1). В соответствии с уравнением (3) поляризацию квази-2D-экситона представим в виде

$$
P_{\alpha}^{(2)}(z, \kappa)=\psi(z-h) \sqrt{l_{w}} W_{\alpha}(\kappa) .
$$

Здесь $W_{\alpha}$ - комплексные амплитуды той же размерности, что $V_{\mp}$ в формуле (13), $l_{w}$ - характерная ширина. Подставим (6), (18) и функции Грина (B11)-(B17) в уравнение (9) с $\mathbf{P}^{(1)}=0$ и выполним интегрирование. $\mathrm{B}$ результате для амплитуд $W_{\alpha}$ при наличии внешней поляризации (6) получаем систему уравнений

$$
\left.\begin{array}{c}
{\left[\omega_{0}^{2}(\kappa)-\Delta \omega_{x x}^{2}(\kappa)-\omega^{2}-i \omega \Gamma\right] W_{x}-\Delta \omega_{x z}^{2}(\kappa) W_{z}=\Omega_{(x)}^{2} C p_{+}^{0},} \\
-\Delta \omega_{z x}^{2}(\kappa) W_{x}+\left[\omega_{0}^{2}(\kappa)-\Delta \omega_{z z}^{2}(\kappa)-\omega^{2}\right. \\
-i \omega \Gamma] W_{z}=i \Omega_{(z)}^{2} C p_{+}^{0} .
\end{array}\right\}
$$

Здесь $\omega_{0}(\kappa)-$ частота экситона квантовой ямы из (3), а поправки

$$
\Delta \omega_{\alpha \beta}^{2}(\kappa)=\Omega_{(\alpha)}^{2}\left(\delta_{\alpha \beta} M_{\alpha \alpha}^{0}+\Delta M_{\alpha \beta}\right)
$$

обусловлены дейсвием собственного электрического поля экситонной поляризации. Величины $M_{\alpha \alpha}^{0}$ и $\Delta M_{\alpha \beta}$ связаны с двумя вкладами в функции Грина $g_{\alpha \beta}=g_{\alpha \beta}^{0}+\Delta g_{\alpha \beta}$ согласно формуле (В20) из Приложения В. Ненулевые матричные элементы

$$
\begin{aligned}
M_{x x}^{0} & =-\frac{2 \pi \kappa}{\varepsilon_{b}} \iint d z d z^{\prime} \psi(z) e^{-\kappa\left|z-z^{\prime}\right|} \psi\left(z^{\prime}\right) \\
& =-M_{z z}^{0}-\frac{4 \pi}{\varepsilon_{b}} \int d z \psi^{2}(z)
\end{aligned}
$$

определяют кулоновские сдвиги $-\Omega_{(\alpha)}^{2} M_{\alpha \alpha}^{0}$ частоты квази-2D-экситона в однородной среде с фоновой проницаемостью $\varepsilon_{b}$. Элементы

$$
\Delta M_{\alpha \beta}(\kappa)=\iint d z d z^{\prime} \psi(z-h) \Delta g_{\alpha \beta}\left(z, z^{\prime}\right) \psi\left(z^{\prime}-h\right)
$$

обусловлены наличием слоя с проницаемостью $\varepsilon_{\infty} \neq \varepsilon_{b}$. С учетом функций Грина (B11)-(B15) из Приложения B 
имеем

$$
\left.\begin{array}{l}
\Delta M_{x x}=i \Delta M_{x z}=-i \Delta M_{z x}=\Delta M_{z z}, \\
\Delta M_{x x}(\kappa)=-4 \pi \frac{\varepsilon_{b}^{2}-\varepsilon_{\infty}^{2}}{\varepsilon_{b}} \frac{1}{\Delta_{-}(\kappa) \Delta_{+}(\kappa)} \frac{e^{-2 \kappa h}}{1-e^{-2 \kappa L}} \kappa I_{c}^{2}(\kappa) .
\end{array}\right\}
$$

В правой части уравнений (19) стоит $p_{+}^{0}$ из (16) и

$$
C(\kappa)=4 \pi \varepsilon_{\infty} \frac{\kappa I_{c}}{\sqrt{l_{w}}} \frac{1}{\Delta_{-} \Delta_{+} \operatorname{sh}(\kappa L)} e^{-\kappa\left(\left|z_{0}\right|+h-L\right)} .
$$

Значение интеграла

$$
I_{c}(\kappa)=\int d z e^{-\kappa z} \psi(z)=\int d z \operatorname{ch}(\kappa z) \psi(z)
$$

от функции $\psi(z)=\psi(-z)$ основного экситонного состояния дается формулой (A3) в Приложении А.

Корни определителя системы уравнений (19) при $\Delta \omega_{\alpha \beta}=0$ дают компоненты $\omega_{L}^{2}=\omega_{0}^{2}-\Omega_{(x)}^{2} M_{x x}^{0}$ и $\omega_{Z}^{2}=\omega_{0}^{2}-\Omega_{(z)}^{2} M_{z z}^{0} \quad$ кулоновского расщепления частоты $\omega_{0}$ квази-2D-экситона в среде с однородной фоновой проницаемостью $\varepsilon_{b}$. В терминах работы [33] $\omega_{L}$ и $\omega_{Z}$ - частоты ортогональных мод $L$ и $Z$, поляризованных соответственно вдоль вектора $\boldsymbol{\kappa}=\kappa \mathbf{e}_{x}$ и по нормали к яме. Нарушение однородности диэлектрического фона пленкой металла $\left(\Delta M_{\alpha \beta} \neq 0\right)$ приводит согласно (19) к взаимодействию между $L$ и $Z$ модами (влияние неоднородного диэлектрического фона на спектр экситонных поляритонов квантовой ямы исследовалось в работе [38]).

\section{5. Уравнения движения для плазмон-экситонов}

В уравнениях (8) и (9) перенесем в левую часть члены с плазмонной $\mathbf{P}^{(1)}$ и экситонной $\mathbf{P}^{(2)}$ поляризациями и с учетом выражений (2), (3), (6) и (7) выполним те же преобразования, что в разд. 4.1 и 4.2. Это дает для амплитуд $V_{-}, V_{+}$плазмонных и $W_{x}, W_{z}$ экситонных мод систему четырех алгебраических уравнений, которая представлена в Приложении С. Из этой системы выделим два уравнения, учитывающие резонансное взаимодействие между плазмонной $V_{-}$и экситонной $W_{x}$ модами, которые поляризованы преимущественно вдоль $\boldsymbol{\kappa}=\kappa \mathbf{e}_{x}$. Плазмоны выбранной квазипродольной моды $V_{-}$ортогональны плазмонам квазипоперечной моды $V_{+}$, к тому же спектры $\omega_{-}(\kappa)$ и $\omega_{+}(\kappa)$ этих мод (ветви 1 и 2 на рис. 2) существенно различаются. Для экситонов полагаем $P_{\alpha}^{(2)}=\delta_{\alpha x} P_{x}^{(2)}, \omega_{x x}(\kappa)=\omega_{0}$, $\Omega_{(\alpha)}=\delta_{\alpha x} \Omega$ и $\Omega^{2}=\omega_{0} \Gamma_{e}$, при этом $W_{z}=0$. Резонансу $\omega_{-}(\kappa)=\omega_{0}$ между плазмоном и квази-2D-экситоном на рис. 2 соответствует точка пересечения дисперсионных ветвей 1 и 3 в области, где $\kappa \sim 1 / L \gg k_{0}$. В резонансе волна поляризации плазмона, имеющая закон дисперсии $\omega_{-}(\kappa)$ и амплитуду $Q_{1}=V_{-}$, взаимодействует с
квази-2D-экситоном, который имеет спектр $\omega_{x}(\kappa)=\omega_{0}$ и амплитуду $Q_{2}=W_{x}$.

Упрощение системы уравнений (C1) из Приложения С применительно к сформулированной модели взаимодействия плазмона и квази-2D-экситона $e 1-h h 1$ дает

$$
\left.\begin{array}{l}
{\left[\omega_{-}^{2}(\kappa)-\omega^{2}-i \omega \gamma\right] Q_{1}+\omega_{-}^{2}(\kappa) u_{1} Q_{2}=\omega_{-}^{2}(\kappa) u_{3} p_{+}^{0}(\omega),} \\
\Omega^{2} u_{2} Q_{1}+\left[\omega_{0}^{2}-\omega^{2}-i \omega \Gamma\right] Q_{2}=\Omega^{2} u_{4} p_{+}^{0}(\omega) .
\end{array}\right\}
$$

Эта система уравнений описывает возбуждение (вынужденные колебания) связанных плазмонного и экситонного гармонических осцилляторов. В уравнения (26) входят множители

$$
\begin{gathered}
u_{1}=\frac{\kappa I_{c} \sqrt{l_{w}}}{2 \operatorname{sh}(\kappa L / 2)} e^{-\kappa(h-L / 2)}, \\
u_{2}=4 \pi \frac{I_{c}}{\sqrt{l_{w}}} \frac{\operatorname{ch}(\kappa L / 2)}{\Delta_{-}} e^{-\kappa(h-L / 2)}, \\
u_{3}=-\frac{\kappa}{2 \operatorname{sh}(\kappa L / 2)} e^{-\kappa\left(\left|z_{0}\right|-L / 2\right)}, \\
u_{4}=4 \pi \frac{\kappa I_{c}}{\sqrt{l_{w}}} \frac{\varepsilon_{\infty}}{\Delta_{-} \Delta_{+} \operatorname{sh}(\kappa L)} e^{-\kappa\left(\left|z_{0}\right|+h-L\right)},
\end{gathered}
$$

$p_{+}^{0}$ из выражения (16) и $I_{c}$ из (25). При наличии внешнего осциллятора $\left(p_{+}^{0} \neq 0\right)$ и затухания парциальных возбуждений (выполнено условие $\operatorname{det} D(\omega, \kappa) \neq 0$ ) система уравнений (26) имеет решение

$$
\begin{array}{r}
Q_{1}=\omega_{-}^{2}(\kappa) \frac{\left(\omega_{0}^{2}-\omega^{2}-i \omega \Gamma\right) u_{3}-\Omega^{2} u_{1} u_{4}}{D(\omega, \kappa)} p_{+}^{0}, \\
Q_{2}=\Omega^{2} \frac{\left(\omega_{-}^{2}(\kappa)-\omega^{2}-i \omega \gamma\right) u_{4}-\omega_{-}^{2}(\kappa) u_{2} u_{3}}{D(\omega, \kappa)} p_{+}^{0},
\end{array}
$$

определяющее плазмонный и экситонный вклады в возбуждаемые плазмон-экситонные моды на частоте внешнего диполя. Здесь

$$
\begin{aligned}
D(\omega, \kappa)= & {\left[\omega_{-}^{2}(\kappa)-\omega^{2}-i \omega \gamma\right] \cdot\left[\omega_{0}^{2}-\omega^{2}-i \omega \Gamma\right] } \\
& -\omega_{-}^{2}(\kappa) \Omega^{2} u_{1} u_{2}
\end{aligned}
$$

- определитель матрицы, стоящей в левой части системы уравнений (26). Таким образом, мы получили в нулевом приближении решение задачи о резонансном взаимодействии мод $Q_{1}$ плазмонов и $Q_{2}$ экситонов во внешнем поле.

\section{6. Нормальные моды плазмон-экситонов}

Обычно спектр смешанных мод обсуждают в пренебрежении затуханием парциальных возбуждений, что дает гармонические нормальные моды. В нашем случае это означает, что при взаимодействии парциальных мод 

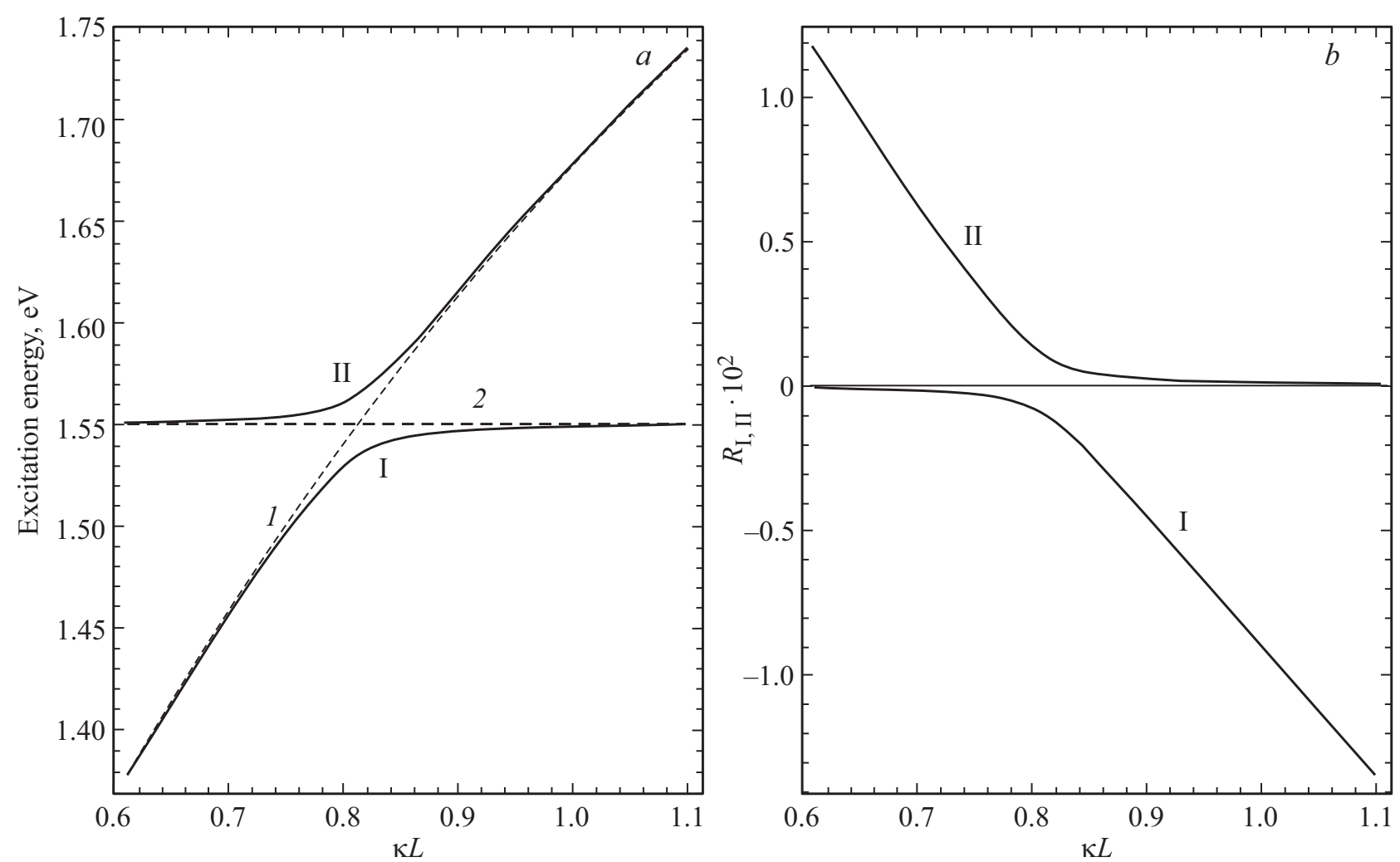

Pис. 3. (a) Собственные энергии $\hbar \omega_{\text {I }}$ (I) и $\hbar \omega_{\text {II }}$ (II) плазмон-экситонов. Пунктиром показаны энергии парциальных плазмонов пленки $\hbar \omega_{-}(\kappa)(1)$ и экситонов квантовой ямы $\hbar \omega_{0}(2)$. (b) Отношение $R_{\mathrm{I}, \mathrm{II}}$ амплитуды $Q_{2}$ экситонов к амплитуде $Q_{1}$ плазмонов

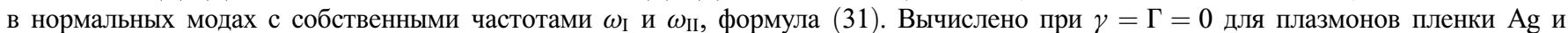
экситонов квантовой ямы GaAs/AlGaAs с $\hbar \Omega=15.7 \mathrm{meV}, l=5 \mathrm{~nm}, h=4 \mathrm{~nm}$ и теми же другими параметрами, что на рис. 2 .

с амплитудами $Q_{1}$ и $Q_{2}$ собственные частоты нормальных мод вычисляются при $\gamma=\Gamma=0$ из уравнения $\left.D(\omega, \kappa)\right|_{\gamma=\Gamma=0}=0$ с определителем (28). В этой идеализированной ситуации для плазмон-экситонных волн, являющихся линейными комбинациями взаимодействующих мод $Q_{1}$ и $Q_{2}$ с заданным $\boldsymbol{\kappa}=\kappa \mathbf{e}_{x}$, находим собственные частоты

$$
\omega_{\mathrm{I}, \mathrm{II}}^{2}(\kappa)=\frac{\omega_{-}^{2}+\omega_{0}^{2}}{2} \mp \sqrt{\frac{\left(\omega_{-}^{2}-\omega_{0}^{2}\right)^{2}}{4}+\Delta \omega^{4}},
$$

где

$$
\Delta \omega^{2}=\omega_{-}(\kappa) \Omega \sqrt{u_{1} u_{2}} .
$$

Из уравнений (26) с $p_{+}^{0}=0$ следует, что отношение амплитуды $Q_{2}^{(\mathrm{I}, \mathrm{II})}$ экситонного вклада к амплитуде $Q_{1}^{(\mathrm{I}, \mathrm{II})}$ плазмонного вклада в плазмон-экситон с частотой $\omega_{\mathrm{I}, \mathrm{II}}(\kappa)$ равно

$$
R_{\mathrm{I}, \mathrm{II}}(\kappa)=\frac{Q_{2}^{(\mathrm{I}, \mathrm{II})}}{Q_{1}^{(\mathrm{I}, \mathrm{II})}}=\frac{\omega_{\mathrm{I}, \mathrm{II}}^{2}-\omega_{-}^{2}}{\omega_{-}^{2} u_{1}}=\frac{\Omega^{2} u_{2}}{\omega_{\mathrm{I}, \mathrm{II}}^{2}-\omega_{0}^{2}} .
$$

Условие $R_{\mathrm{I}, \mathrm{II}}(\kappa) \neq 0$ при $\Delta \omega^{2} \neq 0$ означает, что нормальные моды поляризации с частотами $\omega_{\mathrm{I}, \mathrm{II}}(\kappa)$ содержат вклады и плазмонов, и экситонов, т. е. являются плазмонэкситонами.
Условие резонанса $\omega_{-}(\kappa)=\omega_{0}$ при фиксированной частоте экситона $\omega_{0}$ достигается в результате сдвига частоты плазмона $\omega_{-}(\kappa)$ путем изменения его волнового числа $\kappa$, см. рис. 2. Собственные частоты $\omega_{\mathrm{I}, \mathrm{II}}(\kappa)$ плазмон-экситонов из $(29)$ вблизи резонанса $\omega_{-}(\kappa)=\omega_{0}$ представлены на рис. 3, $a$ в зависимости от параметpa $\kappa L$. На рис. $3, b$ показана зависимость от $\kappa L$ отношения (31) аплитуд парциальных мод, образующих плазмон-экситоны с частотами $\omega_{\mathrm{I}, \mathrm{II}}(\kappa)$. Эти зависимости характеризуют влияние константы связи $\Delta \omega$ из (30) на положение собственных частот $\omega_{\mathrm{I}, \mathrm{II}}(\kappa)$ и на состав $(31)$ нормальных плазмон-экситонных мод при разных $\kappa$. Из выражения (29) следует, что разность собственных частот при $\omega_{-}=\omega_{0}$ составляет $\omega_{\text {II }}-\omega_{\mathrm{I}} \approx \Omega\left(u_{1} u_{2}\right)^{1 / 4}$. При этом, как видно из рис. $3, a$, при всех $\kappa$ парциальные частоты экситонов $\omega_{0}$ и плазмонов $\omega_{-}(\kappa)$ лежат между собственными частотами плазмон-экситонных мод, т. е. $\omega_{\text {I }}<\omega_{0}, \omega_{-}<\omega_{\text {II. }}$ В согласии с [13] можно утверждать, что при любой константе взаимодействия $\Delta \omega$ в области $\left|\omega_{-}-\omega_{0}\right| \ll \Delta \omega$, т.е. в непосредственной близости к резонансу $\omega_{-}=\omega_{0}$, проявляется сильная связанность парциальных возбуждений. Для незатухающих мод она проявляется в качественной перестройке спектра и появлении при $\omega_{-}=\omega_{0}$ биений (периодической перекачки энергии между плазмонами и экситонами за 


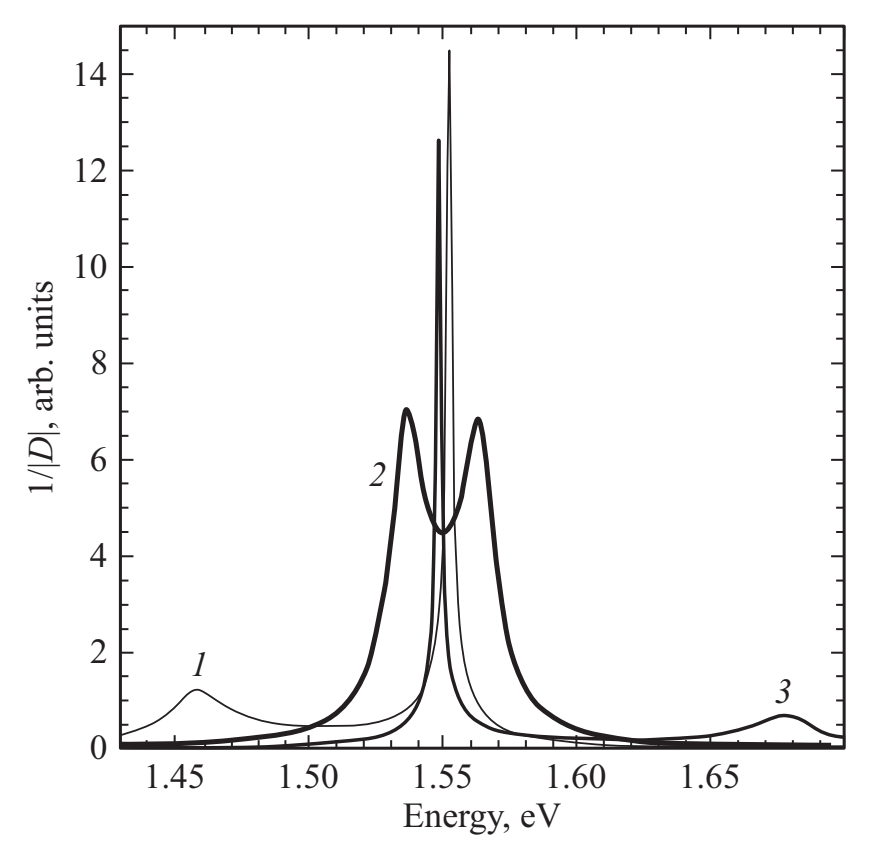

Рис. 4. Зависимость от $\hbar \omega$ спектральной функции плазмонэкситонов $1 /|D(\omega, \kappa)|$ из (28) с параметрами затухания $\hbar \gamma=0.02 \mathrm{eV}$ плазмонов в пленке $\mathrm{Ag}$ и $\hbar \Gamma=1 \mathrm{meV}$ квази-2Dэкситонов в квантовой яме GaAs/AlGaAs. Вычислено при следующих значениях $\kappa L: 0.7$ (1), 0.812 (2), 0.9 (3). Другие параметры те же, что на рис. 3.

время $\sim 1 / \Delta \omega)$, что следует при $\gamma=\Gamma=0$ из уравнений типа (26), зависящих от времени.

Подобный элементарный анализ спектра имеет смысл в случае резонанса между реальными возбуждениями со слабым затуханием и достаточно большой константой связи. Это относится, например, к резонансному вза- имодействию между плазмонами и молекулярными экситонами, для которых характерны достаточно большие силы осцилляторов и высокая добротность резонансных состояний. В альтернативном случае взаимодействия экситонов большого радиуса и плазмонов константа связи (30) может быть сравнительно мала в смысле $\Delta \omega \sim \sqrt{\omega_{0} \Omega}<\gamma$. В этом случае требуется анализировать наблюдаемый оптический спектр плазмон-экситонов, как это было сделано в [27-29].

\section{7. Численный расчет спектра и обсуждение}

Обсудим теперь спектры возбуждения плазмон-экситонов с учетом реальных параметров затухания парциальных возбуждений, которые делают нормальные моды негармоническими (затухающими). Численные оценки сделаем на основе уравнений (26)-(28) применительно к плазмонам в пленке $\mathrm{Ag}$ и квази-2D-экситонам $e 1-h h 1$ тяжелой дырки в квантовой яме GaAs/AlGaAs. В качестве спектральной характеристики волн плазмонэкситонной поляризации возьмем функцию $1 /|D(\omega, \kappa)|$ с определителем (28). Результат ее расчета при некоторых значениях волнового вектора $\kappa$ представлен на рис. 4. Максимумы в спектре $1 /|D(\omega, \kappa)|$ плазмон-экситонов соответствуют их собственным частотам, которые на рис. 3, $a$ в пренебрежении затуханием демонстрируют антипересечение (расталкивание частот) при приближении к резонансу. Такое же поведение характерно для частот на рис. 4 , которые соответствуют двум максимумам функции $1 /|D(\omega, \kappa)|$ при каждом значении $\kappa$. Высота и ширина пиков в спектре $1 /|D(\omega, \kappa)|$ определяются силой осцилляторов и параметрами затухания парциальных мод. Из рис. 4 видно, что спектр $1 /|D(\omega, \kappa)|$ вдали
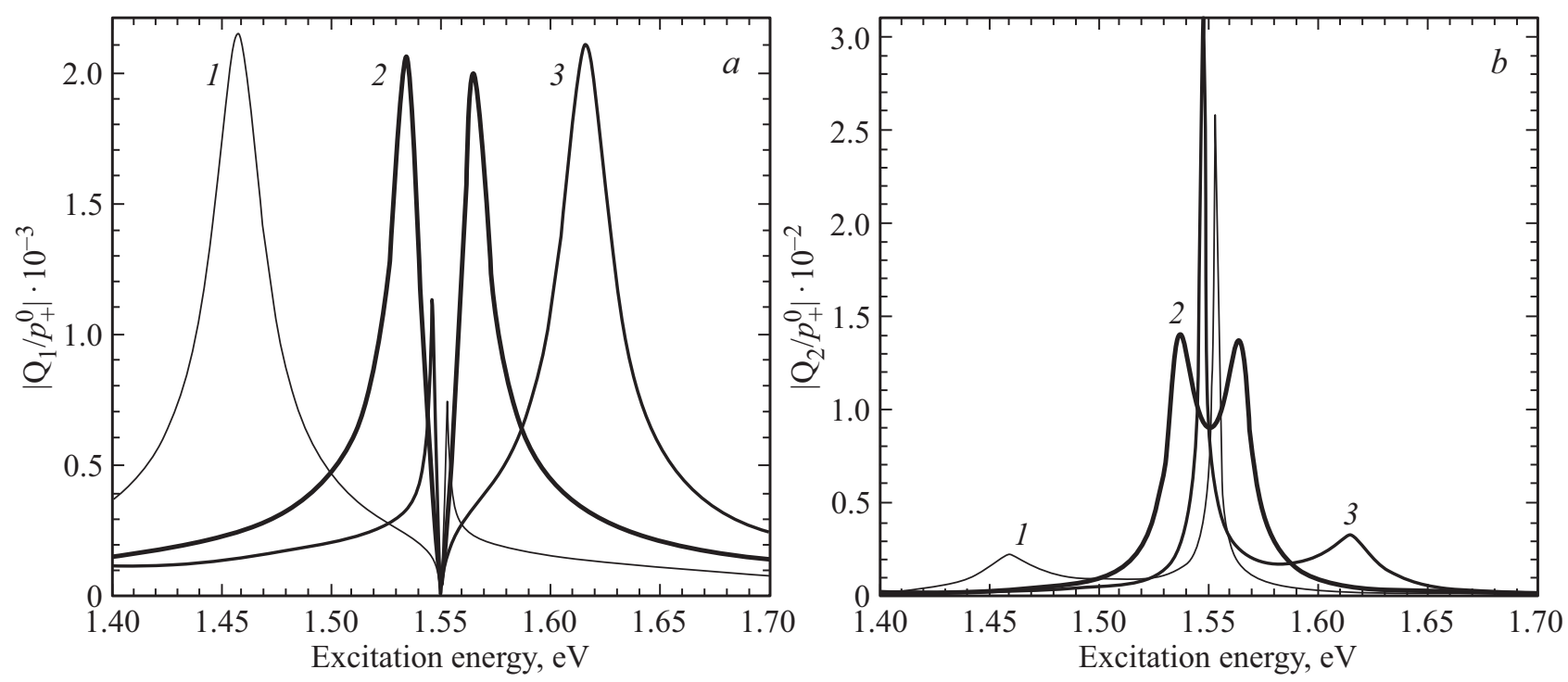

Рис. 5. (a) Плазмонный $\left|Q_{1} / p_{+}^{0}\right|$ и $(b)$ экситонный $\left|Q_{2} / p_{+}^{0}\right|$ вклады в спектры возбуждения плазмон-экситонов в зависимости от $\hbar \omega$, где $\omega$ - частота внешнего дипольного осциллятора $p_{+}^{0}$. Вычислено по формулам (27)-(28) при следующих значениях $\kappa L$ : 0.7 (1), 0.812 (2), 0.9 (3). Другие параметры те же, что на рис. 4. 
от плазмон-экситонного резонанса состоит из узкого экситонного и широкого плазмонного пиков, а при резонансе он трансформируется в два симметричных пика.

На рис. 5 представлены рассчитанные по формулам (26)-(28) спектры стационарного возбуждения парциальных вкладов в плазмон-экситонные моды в зависимости от $\hbar \omega$, где $\omega$ - частота внешнего диполя. Спектры плазмонной амплитуды $\left|Q_{1}(\kappa)\right| /\left|p_{+}^{0}\right|$ показаны на рис. 5, $a$, а спектры экситонной амплитуды $\left|Q_{2}(\kappa) / p_{+}^{0}\right|$ - на рис. $5, b$. Плазмонный и экситонный вклады в спектры возбуждения плазмон-экситонных мод имеют при разных $\kappa$ максимумы на двух частотах, которые испытывают антипересечение вблизи резонанса в согласии с рис. $3, a$.

\section{8. Заключение}

В работе представлена теория нерадиационных плазмон-экситонов, образованных вследствие кулоновского взаимодействия поверхностных плазмонов ультратонкой металлической пленки и низкоразмерных экситонов квантовой ямы. Сформулированы уравнения, описывающие возбуждение плазмон-экситонов в классической модели связанных осцилляторов, роль которых играют поля поляризации квазидвумерных плазмонов и экситонов. Для этих поляризационных возбуждений найдена константа взаимодействия (кулоновской связи), которая обеспечивает возникновение двухпиковой структуры спектра смешанных плазмон-экситонных мод. Поведение частот нормальных мод и спектральных пиков вблизи резонанса между плазмоном и экситоном имеет характерные признаки антипересечения (расталкивания) частот. При учете затухания парциальных мод спектры возбуждения плазмон-экситонов вдали от резонанса состоят из узкого экситонного и широкого плазмонного пиков, которые при резонансе трансформируются в два симметричных пика. Представляется, что полученные результаты несложно обобщить применительно к двумерным плазмонам и экситонам, реализующимся в структурах с атомарно тонкими слоями металла и полупроводника.

Приложение А. Обсудим входящую в соотношение (3) функцию $\psi(z)$ для квантовой ямы шириной $l \ll a_{B}$, где $a_{B}-$ боровский радиус 3D-экситона в материале ямы. Следуя [34,35,37], для внутреннего движения в состоянии $1 s$ квази-2D-экситона возьмем волновую функцию

$$
\Psi_{1 s}\left(\boldsymbol{\rho}, z_{e}, z_{h}\right)=\sqrt{\frac{2}{\pi \tilde{a}^{2}}} e^{-\rho / \tilde{a}} \varphi_{e 1}\left(z_{e}\right) \varphi_{h 1}\left(z_{h}\right),
$$

параметр $\tilde{a}$ которой близок к боровскому радиусу $a_{B}^{2 D}=a_{B} / 2$ двумерного экситона. Поляризация (3) экситона определяется значением функции (A1) при совпадающих координатах электрона и дырки $\rho=0, z_{e}=z_{h}=z$ при этом $\psi(z)=\sqrt{\pi a_{B}^{3}} \Psi_{1 s}(0, z, z)$.
Для модели квантовой ямы с бесконечно высокими стенками для обоих типов носителей $\varphi_{e 1} \varphi_{h 1}=$ $=(2 / l) \cos \left(\pi z_{e} / l\right) \cos \left(\pi z_{h} / l\right) \quad$ в формуле (A1) при $\left|z_{e}\right|,\left|z_{h}\right|<l / 2$. При этом в формуле (4) интеграл

$$
\int d z \psi(z)=\sqrt{l_{e f f}}
$$

определяет эффективную ширину $l_{e f f}=2 a_{B}^{3} / \tilde{a}^{2}>l$. А интеграл (25) равен

$$
I_{c} \equiv \int_{-l / 2}^{l / 2} d z e^{-\kappa z} \psi(z)=\sqrt{l_{e f f}} \frac{4 \pi^{2}}{(\kappa l)^{2}+4 \pi^{2}} \frac{\operatorname{sh}(\kappa l / 2)}{\kappa l / 2}
$$

или $I_{c} \approx \sqrt{l_{e f f}}$ при $\kappa l \approx 1$. Использование величины $l_{e f f} \gg l$ вместо ,физической“ ш ширины квантовой ямы $l$ увеличивает оценки эффектов плазмон-экситонного взаимодействия по сравнению со сделанными в работах [27-29].

Приложение В. Функции Грина. Ниже представлены амплитуды функций Грина $g_{\alpha \beta}\left(z, z^{\prime}, \kappa\right) \exp (i \kappa x)$, вычисленные для двухслойной среды с проницаемостью (1) в пренебрежении электромагнитным запаздыванием. Для функций $g_{\alpha \beta}\left(z, z^{\prime}\right)$ используются обозначения $g_{\alpha \beta}\left(m, m^{\prime}\right)$, где $m$ и $m^{\prime}-$ номера сред $1(z<-L / 2)$, $2(|z|<L / 2)$ и $3(z>L / 2)$, в которых находятся координаты $z$ и $z$. Амплитуды $g_{\alpha \beta}\left(m, m^{\prime}\right)$ для плазмонной задачи $(m=2)$ имеют вид

$$
\begin{gathered}
g_{x x}\left(2,2^{\prime}\right)=-C_{22}\left\{e^{-\kappa\left|z-z^{\prime}\right|}-A\left[\operatorname{ch} \kappa\left(z+z^{\prime}\right)\right.\right. \\
\left.\left.-b \operatorname{ch} \kappa\left(z-z^{\prime}\right)\right]\right\}, \\
g_{z x}\left(2,2^{\prime}\right)=-i C_{22}\left\{e^{-\kappa\left|z-z^{\prime}\right|} \operatorname{sgn}\left(z-z^{\prime}\right)+A\left[\operatorname{sh} \kappa\left(z+z^{\prime}\right)\right.\right. \\
\left.\left.-b \operatorname{sh} \kappa\left(z-z^{\prime}\right)\right]\right\}, \\
g_{x z}\left(2,2^{\prime}\right)=-i C_{22}\left\{e^{-\kappa\left|z-z^{\prime}\right|} \operatorname{sgn}\left(z-z^{\prime}\right)-A\left[\operatorname{sh} \kappa\left(z+z^{\prime}\right)\right.\right. \\
\left.\left.+b \operatorname{sh} \kappa\left(z-z^{\prime}\right)\right]\right\}, \\
g_{z z}\left(2,2^{\prime}\right)=C_{22}\left\{e^{-\kappa\left|z-z^{\prime}\right|}+A\left[\operatorname{ch} \kappa\left(z+z^{\prime}\right)\right.\right. \\
\left.\left.+b \operatorname{ch} \kappa\left(z-z^{\prime}\right)\right]\right\}-\frac{4 \pi}{\varepsilon_{2}} \delta\left(z-z^{\prime}\right), \\
C_{22}=\frac{2 \pi \kappa}{\varepsilon_{2}}, \quad b=\frac{\varepsilon_{1}-\varepsilon_{2}}{\varepsilon_{1}+\varepsilon_{2}} e^{-\kappa L}, \\
g_{x x}\left(2,1^{\prime}\right)=- \\
g_{z x}\left(2,1^{\prime}\right)=-i C_{21}\left(e^{-\kappa z}+b e^{\kappa z}\right) e^{\kappa z^{\prime}}=-i g_{z z}\left(2,1^{\prime}\right), \quad(\mathrm{B} 7) \\
\Delta_{\mp}(\kappa)=\varepsilon_{2}+\varepsilon_{1} \operatorname{th}^{\mp 1}\left(\frac{\kappa L}{2}\right) ; \\
\varepsilon_{1}^{2}-\varepsilon_{2}^{2}
\end{gathered}
$$




$$
\begin{gathered}
C_{21}=\frac{4 \pi \kappa}{\varepsilon_{1}+\varepsilon_{2}} \frac{1}{1-b^{2}}=2 \pi \kappa\left(\varepsilon_{1}+\varepsilon_{2}\right) \frac{e^{\kappa L}}{\operatorname{sh} \kappa L} \frac{1}{\Delta_{-} \Delta_{+}} \\
=C_{23}=C_{32} ; \\
g_{x x}\left(2,3^{\prime}\right)=-C_{23}\left(e^{\kappa z}-b e^{-\kappa z}\right) e^{-\kappa z^{\prime}}=i g_{x z}\left(2,3^{\prime}\right), \\
g_{z x}\left(2,3^{\prime}\right)=i C_{23}\left(e^{\kappa z}+b e^{-\kappa z}\right) e^{-\kappa z^{\prime}}=i g_{z z}\left(2,3^{\prime}\right) .
\end{gathered}
$$

Для экситонной задачи (квантовая яма в среде $m=3$ ) имеем

$$
\begin{gathered}
g_{x x}\left(3,3^{\prime}\right)=-C_{33}\left[e^{-\kappa\left|z-z^{\prime}\right|}+B e^{-\kappa\left(z+z^{\prime}\right)}\right], \\
g_{z x}\left(3,3^{\prime}\right)=-i C_{33}\left[e^{-\kappa\left|z-z^{\prime}\right|} \operatorname{sgn}\left(z-z^{\prime}\right)+B e^{-\kappa\left(z+z^{\prime}\right)}\right], \\
g_{x z}\left(3,3^{\prime}\right)=-i C_{33}\left[e^{-\kappa\left|z-z^{\prime}\right|} \operatorname{sgn}\left(z-z^{\prime}\right)-B e^{-\kappa\left(z+z^{\prime}\right)}\right], \\
g_{z z}\left(3,3^{\prime}\right)=C_{33}\left[e^{-\kappa\left|z-z^{\prime}\right|}-B e^{-\kappa\left(z+z^{\prime}\right)}\right]-\frac{4 \pi}{\varepsilon_{1}} \delta\left(z-z^{\prime}\right), \\
C_{33}=\frac{2 \pi \kappa}{\varepsilon_{1}}, \quad B=\frac{b}{1-b^{2}}\left(e^{2 \kappa L}-1\right)=\frac{\varepsilon_{1}^{2}-\varepsilon_{2}^{2}}{\Delta_{-} \Delta_{+}} \frac{e^{\kappa L}}{\operatorname{sh} \kappa L} ; \\
g_{x x}\left(3,1^{\prime}\right)=-i g_{x z}\left(3,1^{\prime}\right)=-i g_{z x}\left(3,1^{\prime}\right) \\
=-g_{z z}\left(3,1^{\prime}\right)=-C_{31} e^{-\kappa\left(z-z^{\prime}\right)}, \\
C_{31}=-2 \pi \kappa \frac{4 \varepsilon_{2}}{\left(\varepsilon_{1}+\varepsilon_{2}\right)^{2}} \frac{1}{1-b^{2}} ; \quad(\mathrm{B} 16) \\
g_{x x}\left(3,2^{\prime}\right)=-C_{32} e^{-\kappa z}\left(e^{\kappa z^{\prime}}-b e^{-\kappa z^{\prime}}\right)=-i g_{z x}\left(3,2^{\prime}\right), \\
g_{x z}\left(3,2^{\prime}\right)=-i C_{32} e^{-\kappa z}\left(e^{\kappa z^{\prime}}+b e^{-\kappa z^{\prime}}\right)=-i g_{z z}\left(3,2^{\prime}\right) .
\end{gathered}
$$

Функции $g_{\alpha \beta}(m, m)$ с аргументами $z$ и $z^{\prime}$ из одной и той же $m$-й среды представляются в виде

$$
g_{\alpha \beta}\left(z, z^{\prime}\right)=g_{\alpha \beta}^{0}\left(z-z^{\prime}\right)+\Delta g_{\alpha \beta}\left(z, z^{\prime}\right) .
$$

Здесь член $g_{\alpha \beta}^{0}$ связан с поляризацией однородного диэлектрического фона безграничной $m$-й среды, а $\Delta g_{\alpha \beta}$ описывает влияние неоднородности фона.

Приложение С. Подставим (13) и (18) в уравнения (8) и (9) и используем функции Грина из Приложения В. В результате для взаимодействующих плазмонных и экситонных осцилляторов с амплитудами $Q_{1}=V_{-}, Q_{2}=W_{x}, Q_{3}=V_{+}$и $Q_{4}=W_{z}$ получаем уравнения

$$
\left(\hat{H}-\omega^{2} \hat{I}\right) \cdot \mathbf{Q}=\mathbf{F} .
$$

Здесь $\mathbf{Q}$ - вектор с компонентами $Q_{n}, \hat{I}-$ единичная матрица. Элементы матрицы $\hat{H}$ и компоненты вектора $\mathbf{F}$ равны

$$
\begin{gathered}
H_{11}=\omega_{-}^{2}-i \omega \gamma, H_{12}=\frac{\omega_{-}^{2}}{\operatorname{sh}(\kappa L / 2)} A_{0}=i H_{14}, H_{13}=0, \\
H_{21}=\Omega_{(x)}^{2} \frac{\operatorname{ch}(\kappa L / 2)}{\Delta_{-}} A_{1}, \quad H_{22}=\omega_{x x}^{2}-i \omega \Gamma,
\end{gathered}
$$

$$
\begin{gathered}
H_{23}=\Omega_{(x)}^{2} \frac{\operatorname{sh}(\kappa L / 2)}{\Delta_{+}} A_{1}, H_{24}=-\Omega_{(x)}^{2} \Delta M_{x z}=-\Delta \omega_{x z}^{2}, \\
H_{31}=0, H_{32}=\frac{\omega_{+}^{2}}{\operatorname{ch}(\kappa L / 2)} A_{0}=i H_{34}, H_{33}=\omega_{+}^{2}-i \omega \gamma, \\
H_{41}=i \Omega_{(z)}^{2} \frac{\operatorname{ch}(\kappa L / 2)}{\Delta_{-}} A_{1}, H_{42}=-\Omega_{(z)}^{2} \Delta M_{z x}=-\Delta \omega_{z x}^{2}, \\
H_{43}=i \Omega_{(z)}^{2} \frac{\operatorname{sh}(\kappa L / 2)}{\Delta_{+}} A_{1}, \quad H_{44}=\omega_{z z}^{2}-i \omega \Gamma, \\
\left(F_{1}, F_{3}\right)=p_{+}^{0} \frac{\kappa}{2}\left(-\frac{\omega_{-}^{2}}{\operatorname{sh}(\kappa L / 2)}, \frac{\omega_{+}^{2}}{\operatorname{ch}(\kappa L / 2)}\right) e^{-\kappa\left(\left|z_{0}\right|-L / 2\right)}, \\
\quad\left(F_{2}, F_{4}\right)=p_{+}^{0} \kappa\left(\Omega_{(x)}^{2}, i \Omega_{(z)}^{2}\right) A_{2}, \\
A_{0}=\frac{\kappa I_{c} \sqrt{l_{w}}}{2} e^{-\kappa(h-L / 2)}, \quad A_{1}=4 \pi \frac{I_{c}}{\sqrt{l_{w}}} e^{-\kappa(h-L / 2)}, \\
A_{2}=4 \pi \frac{I_{c}}{\sqrt{l_{w}}} \frac{\varepsilon_{2}}{\Delta_{-} \Delta_{+} \operatorname{sh}(\kappa L)} e^{-\kappa(|z 0|+h-L)} .
\end{gathered}
$$

\section{Конфликт интересов}

Автор заявляет об отсутствии конфликта интересов.

\section{Список литературы}

[1] I. Egri. Phys. Rep. 119, 6, 364 (1985).

[2] Поверхностные поляритоны. Электромагнитные волны на поверхностях и границах раздела сред. / Под ред. В.М. Аграновича, Д.Л. Миллса. Наука, М. (1985). 525 с.

[3] A.A. Toropov, T.V. Shubina. Plasmonic Effects in MetalSemiconductor Nanostructures. Oxford Univ. Press (2015). $371 \mathrm{p}$.

[4] W. Cai, V. Shalaev. Optical Metamaterials. Fundamentals and Applications. Springer (2010). $200 \mathrm{p}$.

[5] В.М. Агранович, Ю.В. Конобеев, М.А. Мехтиев. ФТТ 10, 6, 1754 (1968).

[6] В.М. Агранович, М.Д. Галанин. Перенос энергии электронного возбуждения в конденсированных средах. Наука, M. (1978). $383 \mathrm{c}$.

[7] M. Achermann. J. Phys. Chem. Lett. 1, 2837 (2010).

[8] A.O. Govorov, G.W. Bryant, W. Zhang, T. Skeini, J. Lee, N.A. Kotov, J.V. Slocik, R.R. Naik. Nano Lett. 6, 5, 984 (2006).

[9] J.-H. Song, T. Atay, S. Shi, H. Urabe, A.V. Nurmikko. Nano Lett. 5, 8, 1557 (2005).

[10] A. Neogi, C.-W. Lee, H.O. Everitt, T. Kuroda, A. Tackeuchi, E. Yablonovitch. Phys. Rev. B 66, 15, 153305 (2002).

[11] A.A. Toropov, T.V. Shubina, K.G. Belyaev, S.V. Ivanov, P.S. Kop'ev, Y. Ogawa, F. Minami. Phys. Rev. B 84, 8, 085323 (2011).

[12] Y. Fedutik, V.V. Temnov, O. Schops, U. Woggon, M.V. Artemyev. Phys. Rev. Lett. 99, 13, 136802 (2007).

[13] Л.И. Мандельштам. Лекции по теории колебаний. Наука, M. (1972). $466 \mathrm{c.}$

[14] M.R. Philpott. J. Chem. Phys. 62, 1812 (1975).

[15] В.М. Агранович, В.Л. Гинзбург. Кристаллооптика с учетом пространственной дисперсии и теория экситонов. Наука, M. (1979). $432 \mathrm{c}$.

[16] В.А. Кособукин. ЖТФ 56, 8, 1481 (1986). 
[17] J. Bellessa, C. Bonnand, J.C. Plenet, J. Mugnier. Phys. Rev. Lett. 93, 3, 036404 (2004).

[18] Y. Sugawara, T.A. Kelf, J.J. Baumberg, M.E Abdelsalam, P.N. Bartlett. Phys. Rev. Lett. 97, 26, 266808 (2006).

[19] S. Balci. Opt. Lett. 38, 21, 4498 (2013).

[20] B.G. DeLacy, O.D. Miller, C.W. Hsu, Z. Zander, S. Lacey, R. Yagloski, A.W. Fountain, E. Valdes, E. Anquillare, M. Soljacic, S.G. Johnson, J.D. Joannopoulos. Nano Lett. 15, 2588 (2015).

[21] М.Г. Кучеренко, Т.М. Чмерева. Оптика и спектроскопия 125, 2, 165 (2018).

[22] V.M. Agranovich, G.C. La Rocca, F. Bassani. Pure Appl. Opt. 7, 119 (1998).

[23] V.M. Agranovich, Yu.N. Gartstein, M. Litinskaya. Chem. Rev. 111, 5179 (2011).

[24] P. Vasa, R. Pomraenke, S. Schwieger, Yu.I. Mazur, Vas. Kunets, P. Srinivasan, E. Johnson, J.E. Kihm, D.S. Kim, E. Runge, G. Salamo, C. Lienau. Phys. Rev. Lett. 101, 11, 116801 (2008).

[25] B.J. Lawrie, K.-W. Kim, D.P. Norton, R.F. Haglund Jr. Nano Lett. 12, 6152 (2012).

[26] V.I. Sugakov, G.V. Vertsimakha. Phys. Rev. B 81, 23, 235308 (2010).

[27] В.А. Кособукин. ФТТ 57, 7, 1413 (2015).

[28] V.A. Kosobukin. Solid State Commun. 228, 43 (2016).

[29] В.А. Кособукин. ФТТ 59, 5, 972 (2017).

[30] Н.С. Аверкиев, А.В. Коротченков, В.А. Кособукин. ФТП 53, 8, 1063 (2019).

[31] W.G. Teich, G. Mahler. Phys. Status Solidi B 138, 2, 607 (1986).

[32] P.B. Johnson, R.W. Christy. Phys. Rev. B 6, 11, 4370 (1972).

[33] L.C. Andreani, F. Bassani. Phys. Rev. B 41, 11, 7536 (1990).

[34] Е.Л. Ивченко. ФТТ 33, 8, 2388 (1991).

[35] E.L. Ivchenko. Optical spectroscopy of semiconductor nanostructures. Alpha Science International Ltd. (2005). $427 \mathrm{p}$.

[36] E.L. Ivchenko, V.P. Kochereshko, P.S. Kop'ev, V.A. Kosobukin, I.N. Uraltsev, D.R. Yakovlev. Solid State Commun. 70, 5, 529 (1989).

[37] E.S. Khramtsov, P.A. Belov, P.S. Grigoryev, I.V. Ignatiev, S.Yu. Verbin, Yu.P. Efimov, S.A. Eliseev, V.A. Lovtcius, V.V. Petrov, S.L. Yakovlev. J. Appl. Phys. 119, 18, 184301 (2016).

[38] В.А. Кособукин. ФТТ 45, 4, 701 (2003).

Редактор Ю.Э. Китаев 